\title{
Participación económica femenina y emprendimiento en el Estado de Nayarit, México
}

\section{Elizabeth Guadalupe Chong González* Yosilin Pérez Pina**}

Fecha de recibido: 8 de agosto de 2017 Fecha de aprobado: 7 de mayo de 2018

Para citar: Chong González, E. G., \& Pérez Pina, Y. (2019). Participación económica femenina y emprendimiento en el Estado de Nayarit, México. Universidad \& Empresa, 21(36), 36-60. DOI: http://dx.doi.org/10.12804/revistas.urosario.edu.co/empresa/a.5988

Doctora en Ciencias por la Universidad Autónoma del Estado de México, profesora-investigadora de la Universidad Politécnica del Valle de Toluca. Correo electrónico: elizagu_80@yahoo.com

** Estudiante de la licenciatura en Gestión Empresarial por el Instituto Tecnológico del Norte de Nayarit, México. Correo electrónico: yosilinnperez@gmail.com 


\section{Resumen}

Con base en información estadística de los censos y del Global Entrepreneurship Monitor (GEM), el objetivo principal del trabajo es dar cuenta de las actividades económicas que desarrolla la población ocupada femenina de Nayarit e identificar los cambios que presentan en su mercado de trabajo, con la finalidad de conocer las principales ocupaciones que desarrollan, su situación ocupacional, así como su participación en la actividad emprendedora. Los resultados muestran que entre 1970 y 1990 las mujeres participaban considerablemente en actividades relativas al sector primario, sin embargo han tomado mayor relevancia las actividades del sector terciario. En lo referente a su situación en el trabajo, en su mayoría son asalariadas y trabajadoras por cuenta propia, en algunos casos estas últimas han fortalecido su negocio convirtiéndose así en empleadoras, destacando el emprendimiento como uno de los principales cambios en el mercado de trabajo.

Palabras clave: mercado de trabajo, población ocupada, participación económica femenina, actividades por cuenta propia y emprendimiento.

\section{Female Economic Participation and Entrepreneurship in the State of Nayarit, Mexico Abstract}

Based on statistical information from the censuses and the Global Entrepreneurship Monitor (GEM), the main objective of this work is to know the economic activities developed by the female population of Nayarit; likewise it is to identify the changes they present in their labor market, in order to know the main occupations that they develop, their occupational situation, as well as their participation in the entrepreneurial activity. The results indicate that between 1970 and 1990, women participated significantly in activities related to the primary sector, however the activities of the tertiary sector have become more relevant. With regard to the situation at work, the women of the study were mostly salaried and self-employed workers, in some cases the latter have strengthened their business, thus becoming employers, a fact that emphases entrepreneurship as one of the changes in the current labor market.

Keywords: Labor market, employed population, women's economic participation, self-employment and entrepreneurship.

\section{Participação econômica feminina e empreendimento no Estado de Nayarit, México \\ Resumo}

Com base em informação estatística dos censos e do Global Entrepreneurship Monitor (GEM), o objetivo principal do trabalho é dar conta das atividades econômicas que desenvolve a população ocupada feminina de Nayarit; igualmente, identificar as mudanças que apresentam em seu mercado de trabalho, com a finalidade de conhecer as principais ocupações que desenvolvem, sua situação ocupacional, assim como a sua participação na atividade empreendedora. Os resultados mostram que entre 1970 e 1990 as mulheres participavam consideravelmente em atividades relativas ao setor primário, no entanto têm tomado maior relevância as atividades do setor terciário. No referente a situação no trabalho, a maioria são assalariadas e trabalhadoras por conta própria, em alguns casos estas últimas tem fortalecido o seu negócio convertendo-se assim em empregadoras, destacando o empreendimento como umas das principais mudanças no mercado de trabalho.

Palavras-chave: mercado de trabalho, população ocupada, participação econômica feminina, atividades por conta própria e empreendimento. 


\section{Introducción}

En la antigüedad, la mujer no se tomaba en cuenta para ningún suceso o decisión, sin importar el ámbito político, económico, cultural o social; poco a poco las mujeres, con trabajo y esfuerzo, han logrado ocupar espacios económicos, políticos, sociales y culturales que solo estaban destinados para el sexo opuesto (Gonzalbo, 1998).

Hoy en día la situación económica del país afecta de manera directa los hogares, por lo que cada vez son más las mujeres que buscan la manera de incorporarse al mercado laboral, en distintas actividades, como servicios domésticos y personales, y el comercio minorista -en algunos casos sin contrato ni derechos laborales-. Es importante mencionar que las mujeres aportan de manera significativa a la economía, ya sea en empresas, en el campo, como emprendedoras o en el trabajo de cuidados no remunerado (Arispe, 2005).

Esta investigación tiene por objetivo dar cuenta de los cambios en el mercado de trabajo de la población femenina económicamente activa en el estado de Nayarit. En primer lugar, se abordan los aspectos teóricos relativos a los cambios en el mercado de trabajo, así como la participación económica femenina; en segundo lugar, se muestran las principales actividades económicas que ha desarrollado la mujer Nayarita, así como su situación en el trabajo, los sectores en los que ha participado y sus tasas de participación, en el periodo comprendido entre 1970 y 2015; finalmente, se aborda el emprendimiento femenino en el estado de Nayarit, su comportamiento y se mencionan algunos casos de éxito.

\section{Problemática}

La participación económica de las mujeres ha crecido notablemente durante las últimas décadas, como consecuencia del incremento en el nivel de escolaridad, la baja fecundidad y la disminución del poder adquisitivo en los hogares; sin embargo, sus condiciones laborales no han mejorado, ya que gran parte de ellas se sitúan en los trabajos con las peores condiciones, tanto de jornada laboral, como de prestaciones y salarios. Debido a esto, gran parte de ellas incursiona en el trabajo por cuenta propia, el cual, a pesar de ser precario, al menos les permite combinar sus actividades laborales con las domésticas, permitiendo una flexibilidad mayor en cuanto al horario. 
Este trabajo pretende analizar y dar a conocer cuáles son las actividades económicas que desarrolla la población ocupada femenina de Nayarit, así como identificar los cambios que presentan en su mercado de trabajo, conocer las principales ocupaciones que desarrollan, su situación ocupacional y su participación en la actividad emprendedora, con la finalidad de dar cuenta de las necesidades y expectativas de las mujeres en relación a su actividad laboral.

\section{Análisis}

\subsection{Marco teórico}

\subsubsection{Mercado de trabajo}

Los mercados de trabajo constituyen el espacio donde se establecen las relaciones productivas y sociales de un país, además de que son parte fundamental de su economía; actualmente, ya no sólo se requiere gente estudiosa, sino con ciertas cualidades, como la creatividad, el profesionalismo y la competitividad (Gutiérrez, 1999).

El mercado de trabajo ha sido estudiado desde diferentes perspectivas. Desde el enfoque neoclásico, el análisis del mercado de trabajo se aborda a partir de la interacción de la demanda y oferta de los diferentes tipos de trabajo, y uno de los conceptos para su análisis es el de capital humano, que está a la idea de que las personas dedican mayor o menor tiempo y recursos para adquirir cualificaciones o educación, de modo que acuden con distintas capacidades al mercado de trabajo. En consecuencia, la situación de pobreza y desempleo es derivada, según esta teoría, del inadecuado nivel de capital humano. Para dar solución a estos dos problemas, la teoría sugiere aumentar el capital humano de los individuos, mejorando los niveles educativos (Becker, 1983).

Por otra parte, la teoría institucionalista del mercado dual del trabajo, elaborada por Michael Piore, habla de una estratificación del mercado de trabajo desde una visión keynesiana, donde el peso de las instituciones tiene un papel determinante en la formación de los salarios, la generación de empleos y la organización del trabajo; estas instituciones constituyen la base estructural sobre la cual se organizan los mercados de trabajo. A su vez, esta teoría contempla a la sociedad integrada por dos segmentos principales: el sector primario, caracterizado por unidades productivas con alta concentración de capital, que 
operan con tecnología intensiva en capital y sistemas organizativos modernos, que son los negocios que crean los trabajos calificados. El resultado es la existencia de puestos de trabajo complejos y calificados con trabajadores de un alto perfil profesional que los ejecutan; en consecuencia, en el sector primario se ubican los trabajadores que realizan el trabajo más creativo, calificado y bien remunerado (Piore, 1983; Gutiérrez, 1999). Por su parte, el sector secundario se caracteriza por la existencia de empresas y negocios precarios en capital, que operan con baja tecnología y un uso intensivo de la mano de obra y, en consecuencia, paga salarios bajos; asímismo, por ser empresas muy sensibles a los cambios de la demanda, sólo contratan a trabajadores eventuales.

A partir de los años ochenta, el mercado de trabajo ha sido abordado desde el punto de vista de la flexibilidad y se refiere a la situación de que la mano de obra es fácilmente sustituible y movible dadas sus características de calificación; sin embargo, diversos estudios han demostrado que ésta sólo ha causado precarización en las condiciones de trabajo de los asalariados, debido a que oculta sus condiciones de contratación, salariales, de permanencia y despido (Lara, 2001; García, 2001; Chávez, 2001).

Recientemente, ha aumentado la participación de mujeres y hombres emprendedores en el mercado de trabajo, por lo que existe la necesidad de intentar desarrollar un planteamiento nuevo para estudiarlo, ya que esta situación ha surgido como resultado de un alto crecimiento en la oferta de trabajo, la fuerte precarización salarial y una alta calificación de los trabajadores, por lo que se requiere ver al mercado como un todo, no sólo como aquel en el que existen exclusivamente asalariados.

\subsubsection{Cambios en el mercado de trabajo}

A lo largo del tiempo, el mercado de trabajo ha sufrido diversos cambios; primero, pasó de estar en una sociedad completamente agrícola, donde los trabajos que predominaban principalmente eran enfocados hacia este sector, a una sociedad que, a través de las exportaciones, la eficiencia y la competitividad internacional, transitó hacia la industrialización, donde predominaban el sector manufacturero, la industria maquiladora y la construcción. Finalmente, con el progreso tecnológico se ha propiciado el ahorro de mano de obra y se ve un fuerte incremento de una sociedad donde prevalecen actividades relativas al comercio y al sector servicios (García, 2001; Carton de Grammont, 2009). 
La tabla 1 muestra los cambios en el mercado de trabajo por sector de actividad en México, donde se puede ver esa transición en la ocupación de la población económicamente activa del sector primario al terciario, predominando notablemente los servicios.

Tabla 1. Distribución porcentual de la fuerza de trabajo por rama de actividad, México 1970-2015

\begin{tabular}{lccccc}
\hline Rama de actividad & $\mathbf{1 9 7 0}$ & $\mathbf{1 9 9 0}$ & $\mathbf{2 0 0 0}$ & $\mathbf{2 0 1 0}$ & $\mathbf{2 0 1 5}$ \\
\hline $\begin{array}{l}\text { Agricultura, ganadería, silvicultura, } \\
\text { pesca y caza }\end{array}$ & 39,39 & 22,65 & 16,12 & 13,36 & 11,08 \\
\hline $\begin{array}{l}\text { Minería, industrias manufactureras, } \\
\text { electricidad y agua }\end{array}$ & 18,55 & 20,97 & 19,81 & 16,07 & 16,73 \\
\hline Construcción & 4,41 & 6,82 & 7,87 & 8,38 & 8,43 \\
\hline Comercio & 9,24 & 13,28 & 16,82 & 19,21 & 18,34 \\
\hline $\begin{array}{l}\text { Servicios de transporte, comunicación, } \\
\text { profesionales, financieros, sociales, } \\
\text { gobierno y otros }\end{array}$ & 22,64 & 32,85 & 37,01 & 41,67 & 43,77 \\
\hline $\begin{array}{l}\text { Insuficientemente especificada } \\
\text { Total }\end{array}$ & 5,77 & 3,43 & 2,37 & 1,32 & 1,66 \\
\hline
\end{tabular}

Fuente: INEGI (1970, 1990, 2000, 2010, 2015).

Para el caso de la distribución ocupacional por sexo y por rama de actividad, se observa que no hay gran diferencia, ya que predomina la actividad de servicios para ambos.

La situación en el trabajo de la población ocupada de México no ha cambiado significativamente, pues gran parte de ellos continúan siendo trabajadores asalariados; sin embargo, en los últimos años se ha empezado a ver un incremento en las actividades por cuenta propia y empleadores, como resultado de las escasas oportunidades laborales que ofrece el país y de las altas tasas de población en edad de trabajar cuyo mercado laboral formal no las alcanza a cubrir (ver tabla 2). 
Tabla 2. Distribución porcentual de la población ocupada por situación en el trabajo, México 1970-2015

\begin{tabular}{lccccc}
\hline \multicolumn{1}{c}{ Situación en el trabajo } & $\mathbf{1 9 7 0}$ & $\mathbf{1 9 9 0}$ & $\mathbf{2 0 0 0}$ & $\mathbf{2 0 1 0}$ & $\mathbf{2 0 1 5}$ \\
\hline Trabajadores asalariados* $^{*}$ & 62,18 & 68,09 & 67,31 & 67,63 & 73,16 \\
\hline Empleadores & 6,16 & 2,29 & 2,52 & 2,87 & 3,19 \\
\hline Trabajadores por cuenta propia & 25,14 & 23,36 & 23,03 & 24,19 & 19,61 \\
\hline Trabajadores sin pago & 6,53 & 2,51 & 4,50 & 3,16 & 2,78 \\
\hline No especificado & 0,00 & 3,76 & 2,64 & 2,14 & 1,26 \\
\hline Total & 100,00 & 100,00 & 100,00 & 100,00 & 100,00 \\
\hline
\end{tabular}

*Trabajadores asalariados comprende: obreros, jornaleros, peones o ayudantes.

Fuente: INEGI (1970, 1990, 2000, 2010, 2015).

\subsubsection{Participación económica femenina}

La tasa de participación económica femenina en México sólo ha llegado al 42,2 \%, convirtiéndose en la más baja de América Latina, sin olvidar que es inferior a la de los hombres del país por más de 30 \% (El Financiero, 2017).

A pesar de los significativos progresos logrados en las últimas décadas, en todo el mundo los mercados de trabajo siguen estando divididos por géneros y parece haberse estancado el avance hacia la igualdad de género. La participación femenina en la fuerza laboral se ha mantenido por debajo de la participación masculina; las mujeres realizan la mayor parte de los trabajos no remunerados, y cuando tienen un empleo remunerado están sobrerepresentadas en el sector informal y entre la población pobre; también se ven ante significativas diferencias salariales frente a sus colegas varones. En muchos países, las distorsiones y la discriminación en el mercado laboral restringen las opciones de las mujeres para conseguir un empleo remunerado, y la representación femenina en los altos cargos y entre los empresarios sigue siendo baja (Dollar \& Gatti, 1999).

La mujer aporta grandes cantidades de trabajo no remunerado, como en el cuidado de los niños y las tareas domésticas, que en muchos casos siguen siendo invisibles y no se contabilizan en el PIB (Aguirre et al., 2012); además, sigue habiendo diferencias significativas entre hombres y mujeres en cuanto a las horas de trabajo remuneradas y la participación en el trabajo a tiempo parcial (OIT, 2010). Así mismo, existe una significativa disparidad salarial entre hombres y mujeres, aun dentro de las mismas ocupaciones e incluso si se 
tienen en cuenta las características individuales, como la educación. La disparidad de género en lo que respecta a los ingresos es aún mayor en el caso del empleo por cuenta propia que en el empleo asalariado (OCDE, 2012).

En muchos países, las necesidades básicas insatisfechas y la carencia de derechos limitan el potencial de las mujeres para incorporarse al mercado de trabajo formal o convertirse en empresarias (Banco Mundial, 2011). Durante la fase de desaceleración económica de 2007-2009, las diferencias en el empleo de hombres y mujeres se redujeron en la mayor parte de los países. (OCDE, 2012). Para ofrecer a las mujeres las mismas oportunidades económicas y aprovechar completamente el potencial de la fuerza laboral femenina, lo cual tendría importantes repercusiones en el crecimiento y el bienestar futuros, se requerirá un conjunto integrado de políticas que promuevan y apoyen el empleo femenino (Sen, 2001).

Las estadísticas son muy claras al indicar que la mujer ha estado incursionando cada vez con más fuerza en el mercado de trabajo; en México, por ejemplo, su participación en 1970 era de 17,61 \%, y en la actualidad representa el 33,46 \% (INEGI 1970 y 2015). El trabajo de la mujer, antes reservado al ámbito del hogar, se ha liberado de múltiples obstáculos y hoy se ha incorporado a la par con el trabajo masculino en el mercado de laboral (Gandásegui, 1997) y principalmente en las actividades emprendedoras (ver tabla 3).

Tabla 3. Distribución porcentual de la población ocupada femenina por situación en el trabajo México, 1970-2015

\begin{tabular}{lccccc}
\hline \multicolumn{1}{c}{ Situación en el trabajo } & $\mathbf{1 9 7 0}$ & $\mathbf{1 9 9 0}$ & $\mathbf{2 0 0 0}$ & $\mathbf{2 0 1 0}$ & $\mathbf{2 0 1 5}$ \\
\hline Trabajadores asalariados* & 66,64 & 79,65 & 69,23 & 68,38 & 74,82 \\
\hline Empleadores & 6,78 & 1,58 & 1,69 & 2,06 & 2,60 \\
\hline Trabajadores por cuenta propia & 19,43 & 13,32 & 20,64 & 24,07 & 19,15 \\
\hline Trabajadores sin pago & 7,15 & 1,19 & 5,36 & 3,14 & 1,65 \\
\hline No especificado & 0,00 & 4,26 & 3,08 & 2,35 & 1,78 \\
\hline
\end{tabular}

*Trabajadores asalariados comprende: obreros, jornaleros, peones o ayudantes. Fuente: INEGI (1970, 1990, 2000, 2010, 2015). 


\subsubsection{Emprendimiento femenino}

El emprendimiento tiene un papel determinante en el crecimiento económico, en el proceso de innovación y en la creación de empresas pequeñas y medianas que generan puestos de trabajo (Jaramillo, et. al, 2012). Esto ha propiciado que en muchos países se estén instrumentando medidas de política, como financiamiento y subsidios, para apoyar el emprendimiento o para crear y mejorar el ambiente emprendedor.

Definir la palabra emprendedor ayuda a entender los elementos que la componen y facilita su operacionalización con fines de investigación empírica. Schumpeter (1934) definió al emprendedor como una persona innovadora, que propone y aprovecha los cambios, básicamente en cuatro ámbitos: la introducción de nuevos o mejores productos; nuevos o mejores métodos de producción; la apertura de nuevos mercados y la reorganización del proceso administrativo.

Por su parte, Jaramillo, Escobedo, Morales y Ramos (2012) denominan como emprendedores a las personas que perciben oportunidades en el mercado, valoran el riesgo y gestionan los recursos para crear una empresa. Sampedro y Camarero (2007) definen a un emprendedor como una persona que tiene iniciativa y decisión para emprender un negocio o crear empresas.

El Global Entrepreneurship Monitor (2013) concibe al proceso emprendedor centrado en el individuo, el cual se convierte en el sujeto de estudio, pues él pone en marcha la iniciativa emprendedora (ver tabla 4).

Tabla 4. Fases de la actividad emprendedora

\begin{tabular}{ll}
\hline \multicolumn{1}{c}{ Tipo } & \multicolumn{1}{c}{ Característica } \\
\hline Emprendedoras & $\begin{array}{l}\text { No han pagado sueldos y salarios } \\
\text { por más de tres meses. }\end{array}$ \\
\hline Nuevas empresarias & $\begin{array}{l}\text { Han pagado sueldos o salarios } \\
\text { entre los 3 y los 42 meses. }\end{array}$ \\
\hline Empresarias establecidas & $\begin{array}{l}\text { Han pagado sueldos y salarios por } \\
\text { más de 42 meses. }\end{array}$ \\
\hline
\end{tabular}

Fuente: Global Entrepreneurship Monitor GEM (2013). 
Para este trabajo se van a considerar como emprendedoras a las trabajadoras por cuenta propia y empleadoras, porque cumplen claramente con el perfil emprendedor que han definido diversos autores: tienen iniciativa y asunción del riesgo.

En lo que respecta al emprendimiento femenino, existe una mayor proporción de emprendimientos de mujeres, asociado con un origen de necesidad. Las oportunidades de empleo para las mujeres son más escasas, debido al impacto de la socialización de roles y el peso del trabajo doméstico. Con frecuencia se niegan a las mujeres las posibilidades de ejercer un empleo asalariado a causa de sus responsabilidades familiares, su falta de calificaciones, barreras sociales y culturales o por falta de puestos de trabajo. En este contexto, el ejercicio de una actividad independiente o la creación de una empresa -por lo general una microempresa- son con frecuencia los únicos medios de que disponen las mujeres para obtener ingresos que les permitan garantizar su subsistencia y la de sus hijos. La opción emprendedora representa para gran parte de las mujeres una vía para la subsistencia y la sostenibilidad personal y familiar (Carosio, 2004; Sánchez \& Fuentes, 2013).

Las mujeres microempresarias se han convertido en un actor social en la actividad económica, con un enorme potencial para la generación de ingresos y empleos, la superación de la pobreza y la distribución más equitativa de la riqueza. El acceso al capital, la asistencia técnica, el entrenamiento y la consultoría para el crecimiento son aspectos clave para el desarrollo de las empresarias. Aunque para muchas microempresarias el proyecto laboral no incluye la expansión de sus actividades ni el crecimiento de sus negocios, tienen metas muy claras en cuanto a los ingresos que persiguen, ya que la empresa ocupa un lugar importante en la sobrevivencia de sus familias. El sector comercio resulta ampliamente preferido por los emprendimientos femeninos porque, aunque demanda una dedicación horaria importante, facilita a las mujeres la administración de su hogar y su negocio. Las mujeres empresarias, a diferencia de los hombres empresarios, tienen más peso para dedicarse a actividades empresariales; esto se hace evidente tanto en las motivaciones intrínsecas, tales como la necesidad de independencia, como en las motivaciones trascendentales, entre las que se encuentra la conciliación de la vida familiar y la laboral (Carosio, 2004; Amorós \& Pizarro, 2006; Rodríguez \& Santos, 2008; GEM, 2015). 
En el contexto social, familiar y escolar, las mujeres no son incentivadas a ser empresarias independientes o a iniciar un nuevo negocio, pues se piensa que una empresa dirigida por una mujer es una actividad complementaria, es decir, una extensión de la familia. Las mujeres, además del rol tradicional familiar que les ha imputado la sociedad, están desarrollando con mucha capacidad actividades emprendedoras (Amorós \& Pizarro, 2006; Sampedro \& Camarero, 2007).

Según datos del GEM (2015), el 19 \% de los emprendedores del país son mujeres, un número que se traduce en alrededor de 4 millones de emprendedoras, un incremento significativo si se considera que en 1995 la cifra se colocaba en 2,5 millones. Además, muchas mujeres empleadas formalmente buscan incrementar su ingreso a través de pequeños negocios colaterales. Este tipo de emprendimiento es alentado y soportado por modelos de negocio a través de los cuales las mujeres complementan sus ingresos como distribuidoras, vendedoras independientes, representantes, entre otros, aprovechando sus propias redes de contactos (Heller, 2010).

De acuerdo con las cifras de feminización de los trabajadores independientes por grupos de ocupación principal, la participación femenina es claramente superior en los sectores de servicios profesionales, personales, el comercio y la educación (INEGI, 2015).

\section{Resultados}

\subsection{El mercado de trabajo en Nayarit}

El estado de Nayarit tiene una población de 1.237.861 habitantes y representa un 0,9197\% de la población total del país. Del total de habitantes de Nayarit, 595.050 son mujeres, lo que representa el 48,07 \% de la población. (INEGI, 2015).

Al analizar las actividades económicas que realiza la población ocupada de Nayarit, se encontró que en 1970, las actividades más desarrolladas por los nayaritas fueron la agricultura, la ganadería, la silvicultura, la pesca y la caza con un $58 \%$; posteriormente, se encontraron aquellas al sector servicios (16\%) y, por su parte, las de minería, industria manufacturera, electricidad y agua fueron la tercer actividad más desarrollada en el estado (10\%). 
Para 1990, la ganadería, la silvicultura, la pesca y la caza muestra un descenso; sin embargo, continúa concentrando gran parte de la población ocupada (38\%); en segundo lugar, se encuentran nuevamente las actividades relativas al sector servicios, el cual ha crecido considerablemente, ya que para este año agrupa al $25 \%$ de la población ocupada, y en tercer lugar, se tiene a la minería, industria manufacturera, electricidad y agua con $14 \%$.

En el 2000, la minería, la industria manufacturera, electricidad y agua fue la actividad económica más desarrollada por los Nayaritas con el 28 \%, debido a la entrada de diversas industrias manufactureras y a la construcción de varias presas; en un segundo lugar se posicionaron los servicios de transporte, comunicación, profesionales, financieros, sociales y de gobierno (25\%), dejando la agricultura, la ganadería, la silvicultura, la pesca y la caza en un tercer lugar con un $24 \%$, lo que resaltó la caída del sector primario en Nayarit hasta este periodo, ya que a nivel nacional esto había ocurrido desde 1990.

Para 2010 y 2015, se puede observar que los servicios van a la cabeza como las principales actividades económicas realizadas en Nayarit, con el $44 \%$ y $47 \%$, respectivamente; esto sucede como consecuencia del repunte en el sector turístico con la potencialización de la Riviera Nayarita entre 2005 y 2010. En un segundo y tercer lugar se encuentran el comercio, la agricultura, la ganadería, la silvicultura, la pesca y la caza, representando en promedio un $18 \%$. Cabe destacar en este periodo la caída drástica del sector industrial, ya que a partir de este año sólo representa el $8 \%$.

Por último, en el año 2015 los servicios de transporte, comunicación, profesionales, financieros, sociales y gobierno continúan en el sector de actividad económica más desarrollado con un $47 \%$, dejando al comercio y agricultura, ganadería, silvicultura, pesca y caza, en un segundo lugar representados por un $18 \%$, y en un tercer lugar se encuentra el sector de la construcción con un $9 \%$. 
Tabla 5. Distribución porcentual de la población ocupada por sector de actividad económica, Nayarit 1970- 2015

\begin{tabular}{lccccc}
\hline \multicolumn{1}{c}{ Sector de actividad económica } & $\mathbf{1 9 7 0}$ & $\mathbf{1 9 9 0}$ & $\mathbf{2 0 0 0}$ & $\mathbf{2 0 1 0}$ & $\mathbf{2 0 1 5}$ \\
\hline Agricultura, ganadería, silvicultura, pesca y caza & 58,20 & 38,47 & 23,99 & 19,52 & 17,76 \\
\hline Minería, industrias manufactureras, electricidad y agua & 10,46 & 14,39 & 28,10 & 7,68 & 7,59 \\
\hline Construcción & 2,44 & 6,81 & 7,10 & 10,03 & 8,68 \\
\hline Comercio & 7,25 & 10,71 & 13,87 & 18,69 & 17,72 \\
\hline $\begin{array}{l}\text { Servicios de transporte, comunicación, profesionales, } \\
\text { financieros, sociales, gobierno y otros }\end{array}$ & 15,54 & 25,43 & 25,38 & 44,03 & 47,22 \\
\hline No especificado & 6,11 & 4,19 & 1,56 & 0,04 & 1,02 \\
\hline
\end{tabular}

Fuente: INEGI (1970, 1990, 2000, 2010, 2015).

En lo que se refiere a la población ocupada masculina y femenina en el estado de Nayarit, se encontró que en 1970, 1990 y 2000 la actividad más desarrollada por los hombres era la relativa al sector primario con un $66 \%, 39 \%$ y $35 \%$, respectivamente. Con el paso de los años, es visible la disminución de su participación en esta actividad, cayendo a un 26 \% en 2015. A partir del año 2010 y hasta la fecha, el sector terciario es el más desarrollado por la población masculina; por otra parte, las mujeres se han dedicado como actividad principal al sector terciario, el cual ha ido en aumento a lo largo del periodo de estudio.

Respecto a la situación en el trabajo de los Nayaritas, gran parte de la población es asalariada y los empleadores muestran poca participación, destacando que en 1970 era cuando más nayaritas desempeñaban esta situación, debido a que la mayoría eran pequeños agricultores, sin embargo hay un ligero incremento en 2015. Los trabajadores por cuenta propia tienen una participación considerable, debido al incremento del sector servicios y comercio. Por último, los trabajadores sin pago han ido en descenso, ya que su función principal era apoyar en labores agropecuarias, las cuales han descendido considerablemente.

La situación en el trabajo por sexo muestra que tanto para hombres como mujeres no hay cambios significativos, ya que la gran mayoría son asalariados y trabajadores por cuenta propia (figura 1). 


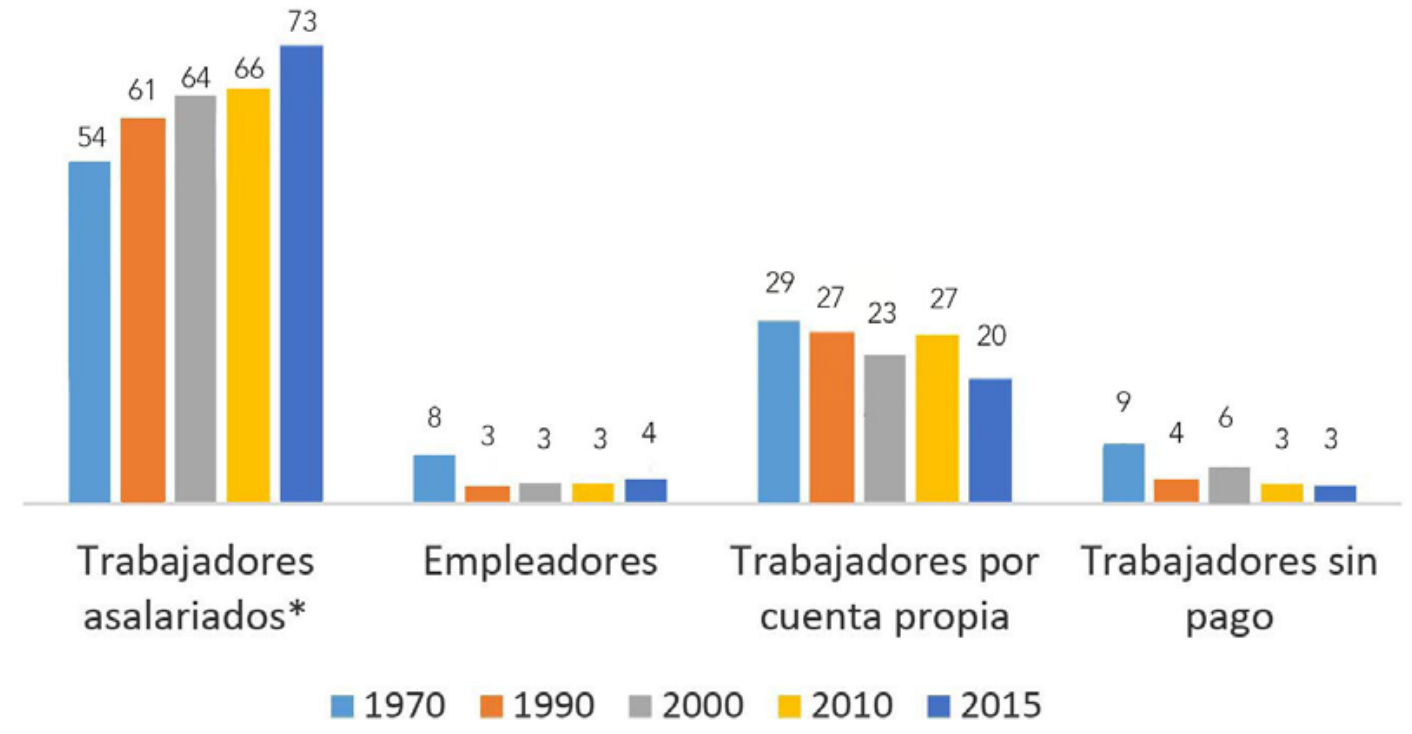

Figura 1. Distribución porcentual de la población ocupada por situación en el trabajo, Nayarit 1970-2015

*Trabajadores asalariados comprende: obreros, jornaleros, peones o ayudantes.

Fuente: INEGI (1970, 1990, 2000, 2010, 2015).

\subsection{Participación económica en Nayarit}

En el periodo comprendido entre 1970 y 1990, las mujeres de Nayarit representaban una cuarta parte de la población ocupada en las actividades agropecuarias y se desempeñaban principalmente como jornaleras. Lo anterior indica la importancia que tenían los mercados de fuerza agricola en la entidad y el peso de la mano de obra femenina en ellos. La estructura productiva de Nayarit estaba basada en las actividades primarias, principalmente en la produccion agricola, y se han observado modificaciones en los cultivos en la región que explican los cambios cualitativos y cuantitativos en los mercados de fuerza de trabajo agrícolas. Tales cambios se reflejaron en la expansión de las relaciones salariales en el campo y en las modificaciones en la composición de la poblacion trabajadora, en la que adquiere importancia la participacion de la población femenina (Barron, 1997).

A fines de los años ochenta, según las encuestas de hogares en el estado (INEGI, 2000), más del $35 \%$ de la PEA eran mujeres, lo que significa 21.500.000 en 1988 y cerca de 25.000.000 al comienzo de los años noventa. Este crecimiento se ha producido tanto en relación con la participación masculina, como en referencia a la propia población femenina 
en edad de trabajar. En 1973 trabajaba un 33 \% de las mujeres mayores de 10 años y en 1988 esa proporción había ascendido al 39 \%. En todo caso, estas tasas de participación económica seguían siendo aún mucho más altas en los hombres, a pesar de que a pesar de que descendieran ligeramente: $77 \%$ en 1973 y $76 \%$ en 1988. Ahora bien, se sabe que la participación laboral femenina del estado sufre de un sobreregistro apreciable, especialmente en los trabajos informales y en las zonas rurales (INEGI, 2010).

Entre 1970 y 1990 la fuerza laboral femenina creció en un 180 \%, mientras que la masculina lo hizo en un 71 \% durante ese mismo período. Por otra parte, la participación laboral de las mujeres según su edad muestra fuertes diferencias con la de los hombres, pues estos comienzan a trabajar a edades más tempranas, especialmente en las zonas rurales. Mientras las mujeres alcanzan su mayor presencia en el mercado de trabajo en torno a los 25 años, los hombres lo hacen pasados los 30, especialmente en las ciudades; estos resultados guardan relación con el hecho de que muchas mujeres desde esa edad se dedican a las actividades del hogar y a la procreación. No obstante, también es importante subrayar que el nivel de actividad no desciende fuertemente, sino hasta después de los 50 años, lo que significa que una proporción apreciable de mujeres no abandona la actividad laboral por razones familiares (INEGI, 2010).

La actividad turística en la costa sur del estado de Nayarit, específicamente en el municipio de Bahía de Banderas, se ha venido fortaleciendo de forma acelerada, dada la prioridad de la política económica a favor del sector servicios, en detrimento del sector agrícola, con lo que se ha redefinido el sector eje de acumulación del capital en la zona, implicando un cambio en el predominio de actividades y de requerimiento de mano de obra en términos cuantitativos y cualitativos, y propiciando otra forma de segmentación de mercados y diferenciación de mano de obra entre sectores. La diversificación de actividades, no sólo se presenta en términos de la amplia variedad de cultivos que se producían en la región, como los de maíz, frijol, sorgo grano, jitomate, arroz, sandía, mango y otros de tipo exótico, sino por la fuerza que adquirieron las actividades relacionadas con el turismo. La necesidad de generar tanto infraestructura básica como complementaria al desarrollo turístico ocasionó que se generaran diversas fuentes de empleo tanto para hombres como para mujeres, que en primer orden compitieron con el mercado laboral agrícola e hicieron que esta región fuera reconocida entre aquellas que tienen los mejores salarios, debido a la escasez relativa de fuerza de trabajo (Sifuentes, 2010). 


\subsubsection{Tasa de participación económica de la población ocupada femenina en Nayarit, 1970-2015}

Las tasas de participación económica femenina van en aumento considerablemente. En 1970 apenas sobrepasaba el $10 \%$, pero conforme pasan los años, las mujeres van haciéndose más visibles en el mercado laboral y en 2015 se encuentran por encima del 35\%. Para el caso masculino, son altas las tasas, pero han permanecido casi constantes a lo largo de todo el periodo de estudio (figura 2).

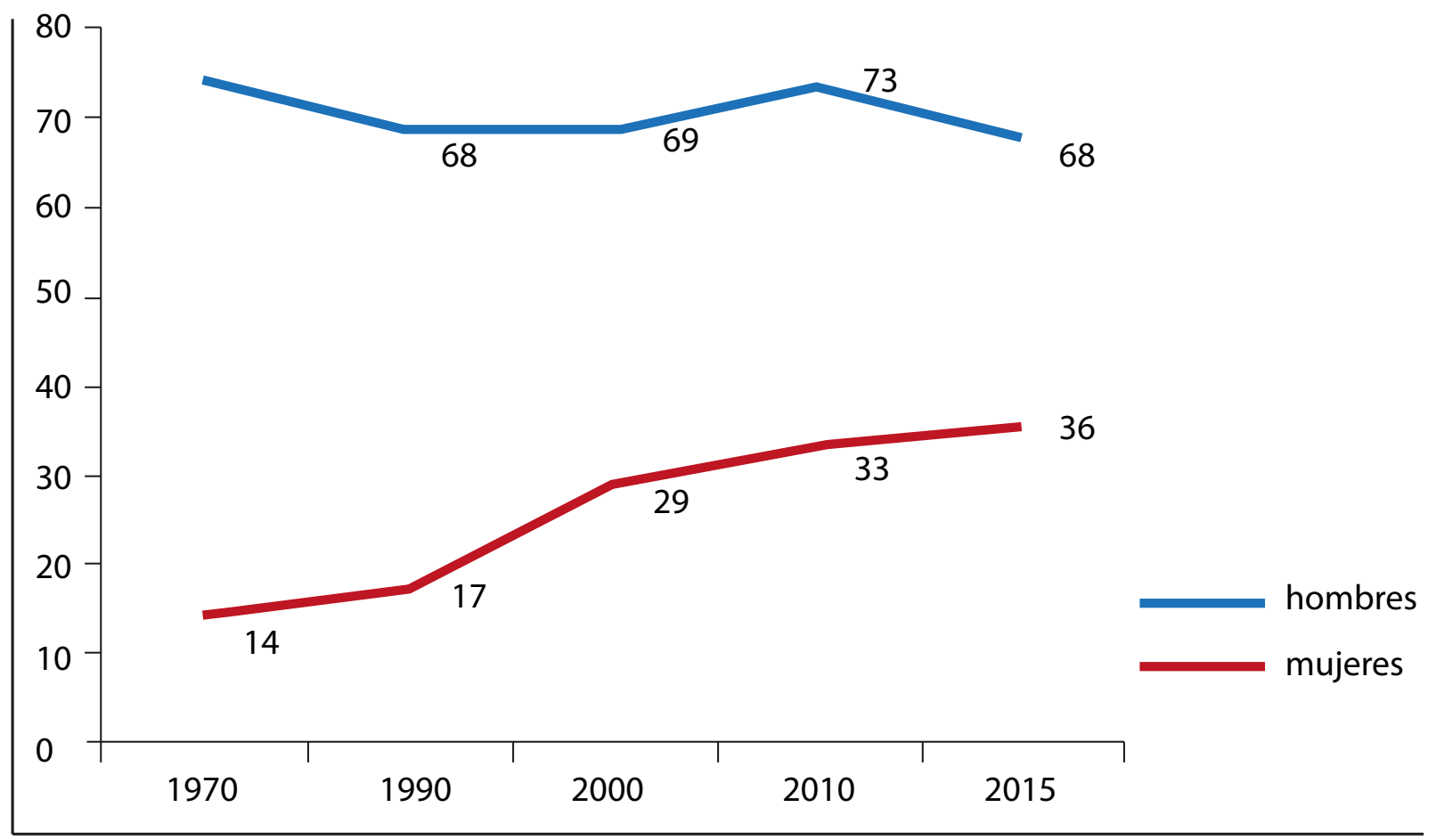

Figura 2. Tasa de participación económica de la población ocupada en Nayarit, 1970-2015 Fuente: INEGI (1970, 1990, 2000, 2010 y 2015).

\subsubsection{Población femenina económicamente activa por ocupación principal en el estado de Nayarit, 1970-2015}

Con base en información del INEGI (2015), respecto a la ocupación principal de las mujeres de Nayarit se encontró que, en el periodo comprendido entre 1970 y 1990, trabajaban en diferentes ocupaciones relativas a labores agropecuarias y de oficina; sin embargo, con el paso de los años las ocupaciones se fueron contrayendo, ubicando a las mujeres en pocas ocupaciones relativas al sector terciario. 
En 1970, las mujeres laboraban primordialmente en actividades agropecuarias y servicios diversos, mientras las comerciantes y vendedoras se colocaban en segundo lugar; así mismo, es importante mencionar que el personal administrativo representaba la tercera posición de las ocupaciones femeninas, y que en ese mismo año las funcionarios superiores y el personal directivo público y privado ocuparon los menores porcentajes. Estos resultados son comprensible ya que en ese año no existían suficientes industrias en el estado.

Para 1990, las mujeres se encontraban ocupadas principalmente como oficinistas (18 \%), comerciantes (17\%) y en el campo de la educación (12\%). A partir de este año sufre una caída drástica el sector primario, por lo que cada vez la población ocupada deja de incorporarse en actividades relativas a ese sector.

En el 2000 continúan dedicándose sustancialmente a actividades de comercio y servicios. Para 2010 se tiene un gran incremento de profesionistas y técnicos, así como de trabajadoras en servicios personales.

En 2015, la principal actividad económica realizada por las mujeres en el estado de Nayarit fue como comerciantes y trabajadoras en servicios diversos, con un $57 \%$; las funcionarias, profesionistas y técnicas administrativas ocuparon el segundo lugar con un $34 \%$, quedando atrás el resto de las ocupaciones con un mínimo de participación.

\subsubsection{Población ocupada femenina por sector de actividad en Nayarit, 1970-2015}

A pesar de su poca intervención en el mercado de trabajo, la mujer ha participado en diversas actividades económicas; sin embargo, en todos los periodos han destacado las actividades pertenecientes al sector terciario, como ocurre también a nivel nacional, cuyo sector es de los preferidos por la población femenina emprendedora, debido a su fácil combinación con las labores domésticas (ver figura 3). 


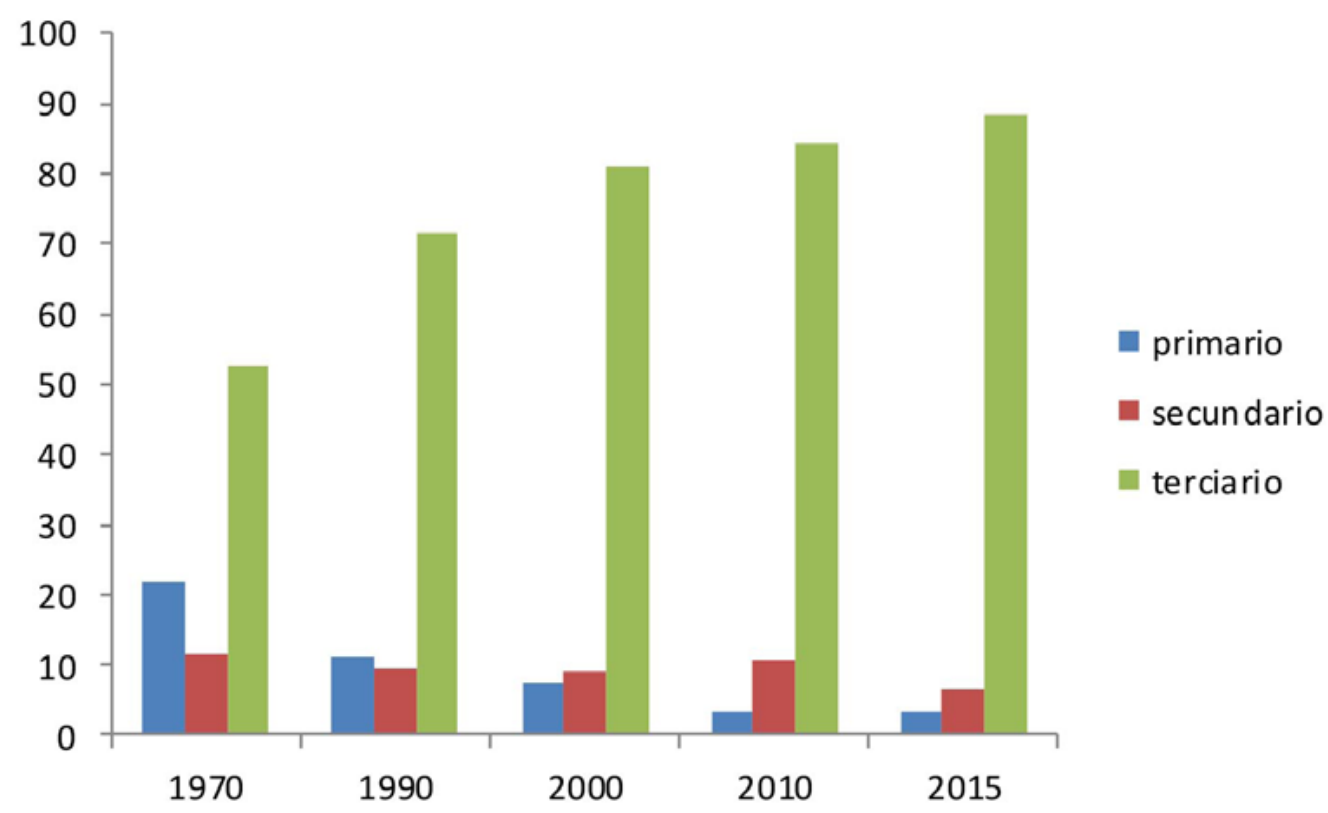

Figura 3. Población femenina ocupada por sector de actividad en Nayarit, 1970-2015 (Porcentaje) Fuente: INEGI (1970, 1990, 2000, 2010 y 2015).

\subsubsection{Población femenina ocupada por situación en el trabajo, Nayarit 1970-2015 (T5)}

En 1970, la posición en el trabajo de las mujeres era como asalariadas con un 56 \%, seguidas de las trabajadoras por cuenta propia con $24 \%$, y en tercer lugar, se encontraban las trabajadoras sin pago, que representaban el $11 \%$. Las empleadoras, por su parte, participaban en un $9 \%$, la cual fue su participación más alta en el periodo comprendido entre 1970 y 2015; esto se debe a que muchas de estas actividades están asociadas con el sector primario, el cual destaca significativamente en este periodo.

Para 1990, la situación en el trabajo de las mujeres en Nayarit continúa siendo en su mayoría asalariada ( $74 \%)$, disminuyen las trabajadoras por cuenta propia, las trabajadoras sin pago y las empleadoras, esto sucede como consecuencia de que muchas de ellas se incorporan como asalariadas en los demás sectores, fuera del sector primario.

A diferencia de los años anteriores, en 2000 y 2010, las mujeres asalariadas disminuyen e incrementan las trabajadoras por cuenta propia. Las empleadoras tienen la misma participación desde 1990 hasta 2010. 
La situación en el trabajo para las mujeres ocupadas en el estado de Nayarit en 2015, se encuentra representada principalmente por las asalariadas, que tuvieron un incremento con respecto a 2000 y 2010 . El porcentaje de mujeres trabajadoras por cuenta propia disminuyó este año, pero el rubro de empleadoras tuvo un incremento, ahora representa el $3 \%$, esto se puede deber a la mayor preparación de las mujeres nayaritas y al apoyo al emprendimiento por parte del gobierno federal y estatal.

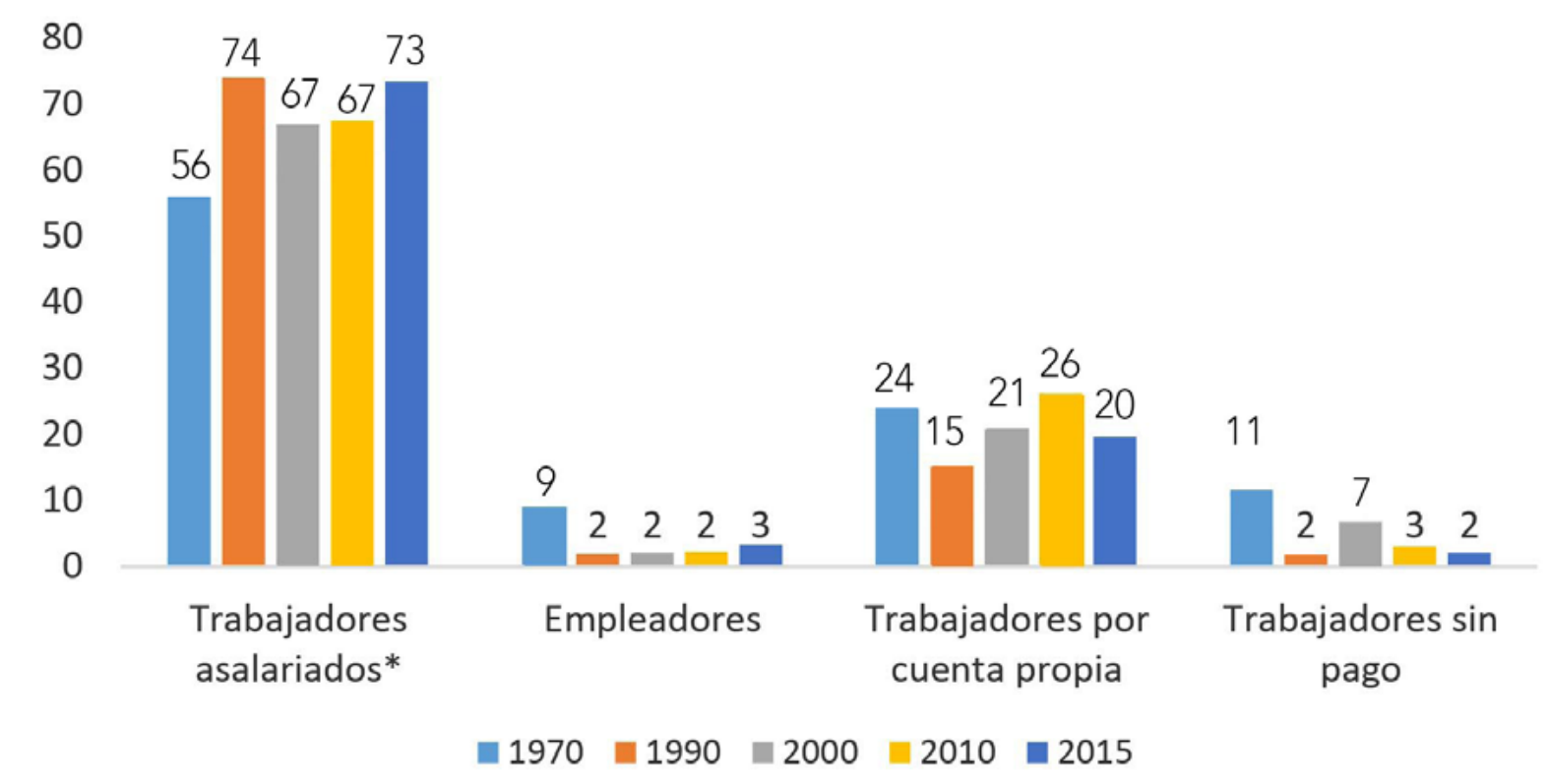

Figura 4. Distribución porcentual de la población ocupada femenina por situación en el trabajo, Nayarit 1970-2015

*Trabajadores asalariados comprende: obreros, jornaleros, peones o ayudantes.

Fuente: INEGI (1970, 1990, 2000, 2010 y 2015).

\subsection{Emprendimiento femenino en Nayarit}

Cuando se habla de crecimiento económico en Nayarit, el emprendimiento es una parte fundamental en los distintos sectores de actividad que se desarollan actualmente en el estado, donde destaca por su capacidad de generar empleos e impulsar la economía.

El rubro de empleadoras y trabajadoras por cuenta propia en Nayarit ha presentado cambios con el paso de los años. En 1970 la participación de las mujeres era muy representativa y estaba enfocada al sector primario principalmente; en 1990 hay un descenso en las actividades por cuenta propia y en el rubro de empleadoras, que se debió principalmente a que la mayoría comenzó a incorporarse en el sector secundario y terciario como asalariadas, y para el periodo comprendido entre 2000 y 2010 las trabajadoras por cuenta propia 
aumentaron debido a las escasas oportunidades laborales que hay tanto a nivel nacional como estatal en el sector formal y las empleadoras se mantienen representando un $2 \%$.

Para 2015 hay una disminución en las trabajadoras por cuenta propia, pero aumentan las empleadoras, esta situación se puede deber a que algunas de las trabajadoras por cuenta propia se trasladaron al rubro de empleadoras, ocasionado por la alta preparación profesional de las mujeres y al incremento en los apoyos a los emprendedores por parte del estado, como son Tepic Emprendedor e Inadem.

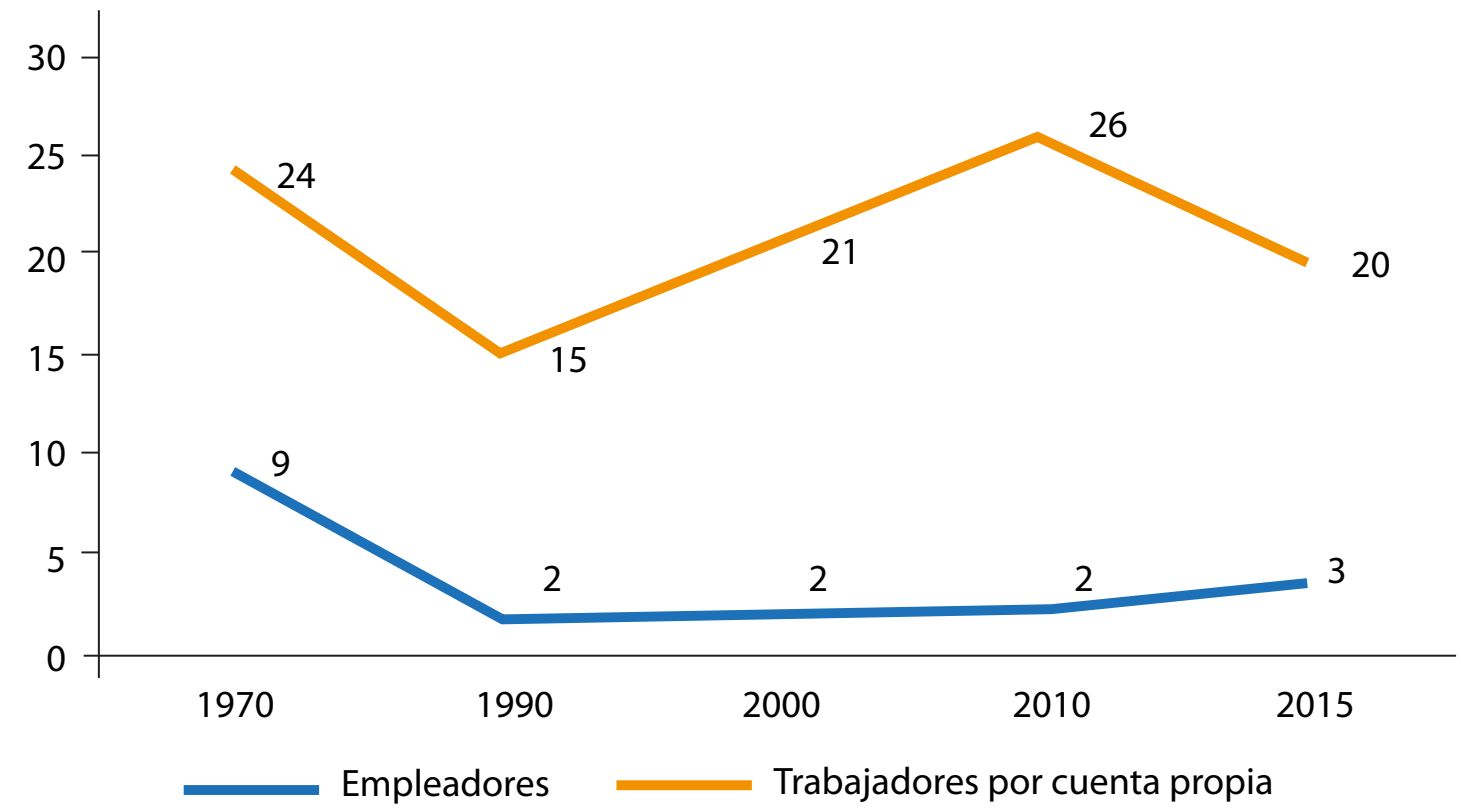

Figura 5. Distribución porcentual de la población emprendedora femenina en el estado de Nayarit, 1970-2015

Fuente: INEGI, 1970, 1990, 200, 2010 y 2015.

Algunos casos de éxito de emprendimiento femenino en el estado de Nayarit se muestran a continuación (López, Gómez \& Haro, 2013):

\section{- Caso 1. Restaurante de mariscos y pescadería "Mariscos Guille"}

Surgió en 2007 y fue la idea de una mujer soltera con dos hijos, que inició con un puesto pequeño de mariscos a bordo de carretera. Era un tejabán con láminas, carecía de muebles, empleados, clientes y lo más importante, dinero. Con el paso de los años sufrió muchas críticas por atender a hombres y por la ubicación del lugar, pero pese a estos obstáculos, Guille continuó con su lucha y hoy en día quien atiende es ella misma con sus hijos y varios empleados. 
Guille, se siente realizada como mujer por haber logrado tanto por sus hijos y se ha dado cuenta también de que es fuente de empleo para muchas personas.

\section{- Caso 2. "Mariscos Silvia"}

En 1991, Silvia Romero trabajaba en una empresa llamada Cooperativa El Pescador, en el área de empaque, en Chilapa, Nayarit. En esta empresa se empaquetaba camarón, el cual despertó un particular interés en ella, ya que observaba las buenas ventas que se obtenían de la empacadora. En 1992 Silvia fue despedida, así que identificó que la mejor opción era invertir el dinero de su liquidación en un negocio familiar en el que se dedicaría a vender mariscos, ya que ella sabía a la perfección qué trato se le tenía que dar a la mercancía.

En la actualidad "Mariscos Silvia" consta de un local totalmente modificado en condiciones perfectas, diez personas al servicio de la empresa y cuatro motocicletas para el servicio a domicilio y la distribución de la mercancía para sus clientes.

\section{- Caso 3. Frutas y verduras}

La microempresa pertenece al sector comercial, se dedica a la venta de frutas y verduras, está ubicada en la comunidad de Sauta, municipio de Santiago Ixcuintla, Nayarit, y fue fundada desde hace más de cinco años por Vanessa García, quien tenía 25 años y comenzó su negocio vendiendo un bajo volumen de frutas y verduras de casa en casa. Ahora ella, que cuenta con estudios de preparatoria en el área económico-administrativo, es la responsable de la administración y el buen funcionamiento de su microempresa.

En la actualidad, su negocio es próspero y se han inculcado valores como el respeto y la honestidad, ya que gracias a ellos esta micro se ha ganado el prestigio de sus clientes, motivo por el cual la dueña sigue buscando más estrategias para que pueda hacer crecer su negocio y así obtener más utilidades, ofrecer empleos y seguir teniendo éxito.

\section{Conclusiones}

El mercado de trabajo en México ha sufrido diversos cambios a lo largo del tiempo, principalmente como resultado de pasar de una economía protegida a una más abierta, la urbanización, el aumento de actividades no agrícolas, el incremento en la escolaridad 
femenina y el descenso en la fecundidad (García, 2001). Esta situación ha impactado de manera negativa a la población ocupada, ya que muchos de ellos se encuentran en trabajos con condiciones laborales precarias; esto afecta aún más a las mujeres, ya que tienen que conciliar sus tareas familiares con las laborales, buscando así actividades por cuenta propia que de manera paulatina les permitan realizar ambas actividades. Por esta razón, es importante que el estado formule estrategias que permitan que ellas consoliden sus negocios y no se quede como una actividad emergente para contrarrestar la falta de ingresos de su hogar o la falta de oportunidades laborales formales en su entorno.

El caso de la población femenina activa en el estado de Nayarit no dista de lo que ocurre a nivel nacional, el cual ha presentado diversos cambios a lo largo del periodo de estudio. Primero, el incremento considerable en sus tasas de participación de $14 \%$ en 1970 a $36 \%$ en 2015; en segundo lugar, el tránsito de actividades del sector primario al terciario, y en tercer lugar su marcada participación en actividades por cuenta propia que, si logran formalizarse, pueden fortalecer su negocio y potencializar su actividad emprendedora.

De manera particular, los tres estudios de caso que se presentan aquí y, en general, la literatura que habla sobre emprendimiento, demuestran que uno de los principales motivos por los cuales las mujeres incursionan en el emprendimiento es la necesidad de sacar adelante a sus familias y de conjugar sus labores familiares con su negocio. Para concluir, es importante resaltar la importancia del sector terciario para las mujeres, donde han encontrado un buen comienzo para desarrollarse económicamente, contribuyendo así a la economía familiar y estatal.

Finalmente, se recomienda a los gobiernos fomentar espacios de guarderías de calidad, en sectores suburbanos y urbanos, para ayudar a las jefas de familia, crear mayores plazas en la administración pública, no por equidad de género, sino por la capacidad de cada persona; también se recomienda dar seguimiento e impulso a las políticas públicas de apoyo y protección a la mujer y por ultimo crear proyectos productivos enfocados a potencializar la actividad emprendedora de las mujeres. 


\section{Referencias}

Aguirre, A., Hoteit, L., Rupp, C., \& Sabbagh, K. (2012). Empowering the Third Billion, Women and the World of Work in 2012. Stuttgart: Booz and Company.

Amorós, J. E., \& Pizarro, O. (2006). Mujeres y dinámica emprendedora: un estudio exploratorio. Universidad y Empresa, 5(11), 15-32.

Arispe, L. (2005). La mujer en el desarrollo de México y de América Latina, México. México: Centro Regional de Investigaciones, Colegio de México.

Banco Mundial. (2011). Informe de Desarrollo Mundial 2012. Igualdad de Género y Desarrollo. Washington: Banco Mundial.

Barrón, A., \& Sifuentes, E. (1997). Mercados de trabajos rurales en México. México: Universidad Nacional Autónoma de México.

Becker, G. (1983). Inversión en capital humano e ingreso. En L. Toharia (Comp.), El mercado de trabajo: teorías y aplicaciones (pp. 39-65). Madrid: Alianza Editorial.

Carosio, A. (2004). Las mujeres y la opción emprendedora. Consideraciones sobre la gestión. Revista Venezolana de estudios de la mujer, 9(23), 79-112.

Carton de Grammont, H. (2009). La degradación del campo mexicano. Revista convergencia, 16(50), 13-55.

Chávez, P. (2001). Flexibilidad en el mercado laboral: orígenes y concepto. Revista Aportes, 6(17), 57-74.

Dollar, D., \& Gatti, R. (1999). Gender Inequality, Income, \& Growth. Are Good Times Good for Women. Washington D.C.: World Bank Gender and Development Working.

El Financiero. (2017). La tasa de participación laboral femenina en México. Recuperado de http://www.elfinanciero.com.mx/economia/mexico-con-la-tasa-de-participacion-laboral-femenina-mas-baja.html

Gandásegui, M. (1997). La segmentación del mercado de trabajo y la discriminación de la mujer. Papeles de población, 3(14), 191-121.

García, B. (2001). Restructuración económica y feminización del mercado de trabajo en México. Revista Papeles de Población, 27(1), 45-61.

Global Entrepreneurship Monitor. (2013). Reporte Nacional de México. Global Entrepreneurship Monitor. Recuperado de http://gemconsortium.org/report

Global Entrepreneurship Monitor. (2015). Reporte Nacional de México. Global Entrepreneurship Monitor. Recuperado de http://gemconsortium.org/report

Gonzalbo, L. (1998). La mujer a través de los años. México: Colegio de México. 
Gutiérrez, E. (1999). Nuevos escenarios en el mercado de trabajo en México 1983-1999. Papeles de población, 5(21), 22-50.

Heller, L. (2010). Mujeres Emprendedoras en América Latina y el Caribe. Realidades, obstáculos y desafios. Santiago de Chile: CEPAL.

Instituto Nacional de Estadística Geografía e Informática. (1990). XI Censo General de Población y Vivienda 1990. Tabulados básicos. Estado de Nayarit: INEGI.

Instituto Nacional de Estadística Geografía e Informática. (2010). Censo de Población y Vivienda 2010. Tabulados básicos. Estado de Nayarit: INEGI.

Instituto Nacional de Estadística Geografía e Informática. (1970). IX Censo General de Población 1970. Estado de Nayarit: Editorial.

Instituto Nacional de Estadística Geografía e Informática. (2000). XII Censo General de Población y Vivienda 2000. Tabulados básicos. Estado de Nayarit: INEGI.

Instituto Nacional de Estadística Geografía e Informática. (2015). Tabulados de la Encuesta Interesal 2015. Estado de Nayarit: INEGI.

Instituto Nacional de Estadística Geografía e Informática. (2015). Ocupación y Empleo. Recuperado de http://www.inegi.org.mx/default.aspx

Jaramillo, J., Escobedo, J., Morales, J., \& Ramos, J. (2012). Perfil emprendedor de los pequeños empresarios agropecuarios en el Valle de Puebla, México. Entramado, 8(1), 44-57.

Lara, S. (2001). Una nueva ruralidad en América Latina. Buenos Aires: Clacso.

López, G.C., Gómez, J.F., \& Haro, J. F. (2013, abril). Historias de éxito de emprendedores con empresas familiares en Nayarit. Ponencia presentada en el XVII Congreso Internacional de Ciencias Administrativas. México.

Morelos, J. (1993). La consistencia interna de los datos corregidos de la población activa censal (1960-1980) y la estimación de las tasas de participación por edad y sexo para 1980. Revista de Estudios Demográficos y Urbanos, 8(2), 307-329.

Organización Internacional del Trabajo. (2010). Women in Labour Markets: Measuring Progress and Identifying Challenges. Ginebra: OIT.

Organización para la Cooperación y el Desarrollo Económicos (OCDE). (2012). The Price of Prejudice: Labour Market Discrimination on the Grounds of Gender and Ethnicity. París: OECD.

Piore, M. (1983). Notas para una teoría de la estratificación del mercado de trabajo. En L. Toharia (Comp.), El mercado de trabajo: teorías y aplicaciones (pp. 125-150). Madrid: Alianza Editorial: Madrid. 
Rendón, T., \& Salas, C. (1986). La población económicamente activa en el censo de 1980: comentarios críticos y una propuesta de ajuste. Revista de Estudios Demográficos y Urbanos, 2(2), 291-309.

Rodríguez, M. J., \& Santos, F. J. (2008). La actividad emprendedora de las mujeres y el proceso de creación de empresa. Revista el Papel de los emprendedores en la economía, 19(841), 117-130.

Sampedro, R., \& Camarero, L. (2007). Mujeres empresarias en la España rural el sujeto pendiente del desarrollo. Revista Internacional de sociología, 65(48), 121-146.

Sánchez, S. M., \& Fuentes, F. (2013). Mujer y Emprendimiento: Un análisis en el contexto universitario español. Revista de Ciencias Sociales, 14(1), 140-153.

Schumpeter, J. A. (1934). The Theory of Economic Development. Cambridge: Harvard University Press.

Sen, A. (2001). Many Faces of Gender Inequality. Nueva Delhi: Frontline.

Sifuentes, E., \& Arteaga, R. (2010). Turismo, migración y cambio en los mercados de trabajo agrícolas. México: Universidad Autónoma de Nayarit. 